\title{
The Two-Axis Linkage System Design of CNC Machine Based on PLC
}

\author{
Qian Wang ${ }^{1, a}$, Zhiyi Huo ${ }^{2}$ \\ 1,2Xingtai Polytechnic College, Xingtai, Hebei, 054035, China \\ ${ }^{a}$ Corresponding author: Qian Wang
}

\begin{abstract}
This essay gives an introduction to the design of control procedures to realize the linkage of axis $\mathrm{X}$ and axis $\mathrm{Y}$ of CNC machine by the use of PLC movement control module, and thereby to accomplish making complicated curved surfaced parts and simulate multi-axis linkage processing environment. This system has developed specially corresponding positioning module point at movement for $\mathrm{NC} \mathrm{X}$ and $\mathrm{Z}$ axis, it can simplify the design process greatly, can improve the machining accuracy and effective. in addition, the control module may reduce the production cost and dilution procedure, at the same time, this system can greatly save operation time and is suitable for wide popularization
\end{abstract}

\section{Introduction}

Single axis machine tool can not meet the processing needs any longer with the advent of complex curved surface parts, as shown in figure 1 . Thereby the machine needs to be improved on the basis of the initial single axis $\mathrm{CNC}$ machine tool to realize two-axis and multi axis in order to fit the changeable requirements of the machining of complex curved surfaces. Processing control process is usually accomplished by relay in the traditional industrial control, while $\mathrm{CNC}$ machine tools need to use $\mathrm{NC}$ program in the process of control. Nowadays, PLC has replaced the relay control in many fields with the development of the micro-electronics technology and computer technology. PLC, as the core control device for industrial application is more and more widely used because of its advantages in the control fields since the 60 's of last century. In this paper the author introduces the positioning module aimed at $\mathrm{X}, \mathrm{Z}$ axis motor of $\mathrm{NC}$ machine tools. This module can simplify the design process greatly and can improve the machining accuracy and effectiveness ${ }^{[1]}$.

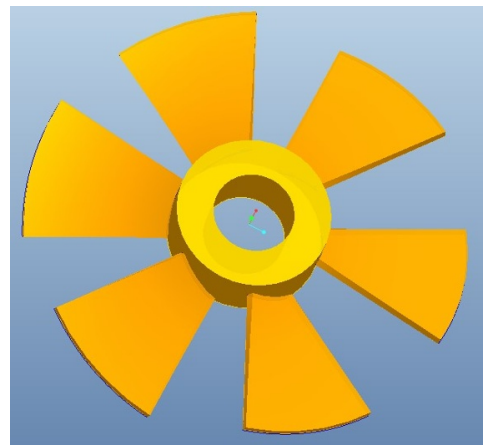

Figure 1 Surface part

a Corresponding author: 39563742@qq.com

\section{Design scheme of two axis system based on PLC}

\subsection{Introduction to PLC control module}

PLC can control the position velocity and acceleration of the linear motion and circular motion by using the special location control module. It can also realize single or multi axis position control, and can combine the motion control and sequence control organically, and finally realize two-axis or multi axis linkage function. This technique has been widely applied in the field of all kinds of general machinery, special machine tools, robots, elevator, automatic transmission line, etc. The positioning control module, as an intelligent $\mathrm{I} / \mathrm{O}$ module, is a microprocessor computer system. It has the information processing function strong and its CPU with PLC can work in parallel, greatly improving the speed and the effect of the control systems ${ }^{[2]}$.

For example, Mitsubishi FX2N-80MT PLC and its special function module of 20GM and HJD-4 experimental platform, The test bench includes required high and low voltage relay contacts, switches, buttons and $\mathrm{CNC}$ machine tool in the control process, Series of FX2N positioning control module of $20 \mathrm{GM}$ can independently control positioning, and it not only has the positioning control language and programming language, but also has the function of command data processing, so it can be independent of more advanced control behavior.

\subsection{The software design}

Program design of $\mathrm{X}, \mathrm{Z}$ axis is for $20 \mathrm{GM}$ system, and the data is transferred to the $20 \mathrm{GM}$ buffer in BMF through the program that PLC sends to, the 20GM system has 
special buffer (BFM\#20 with operation mode synchronous axis and $\mathrm{X}$ axis, BFM\#21with operation mode of $\mathrm{Y}$ axis) for $\mathrm{X}, \mathrm{Z}$ axis motion, and each buffer has two words, and each word represents the different motion characteristics. The 20GM buffer program format is as shown in figure $2^{[3]}$.

$$
\begin{array}{l|l|}
\text { b15 continuous path } \\
\text { b14 } 16 \text { bit command }
\end{array}
$$
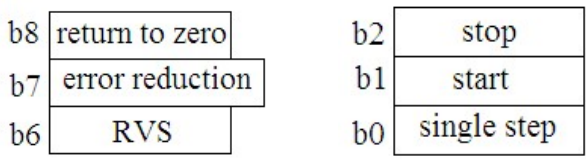

Figure 2. Format of 20GM

\subsection{The hardware design}

The control system principle of two-axis linkage is shown in figure 3; the hardware connection of two axis linkage control system is shown in figure 4. The type of stepper power is 57BYG250E and drive type is SH-20806C as the CNC machine tool. We can automatically switch on QF1 and QF2 when stepper motor, drive control circuit and PLC peripheral KA2 relay and contactor KM2 output circuit is connected, and we can do the following:

(1) The programming on the software "FXGP/WIN-C" may input control program to the PLC through connecting the cable programming to the PLC, the state of the switch PLC to dial "STOP" to programming position;

(2) The positioning software " FXVPS-E" of PC may input code to the 20GM through connecting the cable programming to the 20GM, the state of the switch 20GM to dial "MENU" to manual position;

(3) Contactor KM2 can move PLC when the main contact is closing, and drive $\mathrm{SH}-20806 \mathrm{C}$ is electricity when the state of the PLV switch to the position "RUN";

(4) The state of the switch 20GM to dial to the automatic position "AUTO";

(5) The $X$ axis and $Z$ axis will reset when we press the "reset" button;

(6) Click the "start" button to run program 20GM;

(7) Click the "stop" button to stop running 20GM;

(8) The main contact of the contactor KM2 will be disconnected, and $\mathrm{SH}-20806 \mathrm{C}$ drive will lose power when the bottom left corner of PLC toggle switch to the programming position "STOP" or PLC is in a power down state.

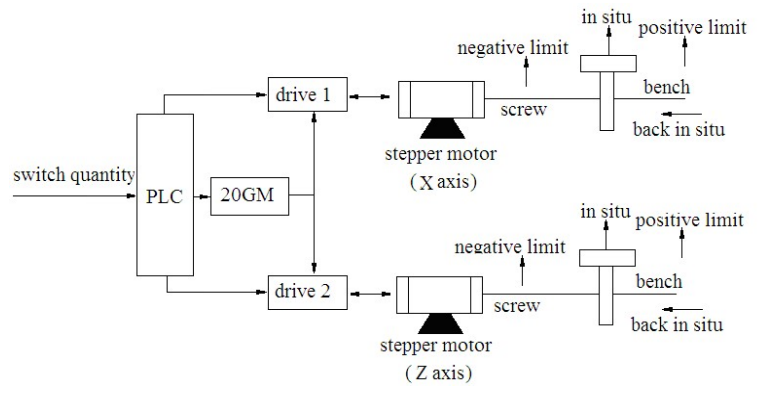

Figure 3 Figure of Two-aixs Coordinated Control

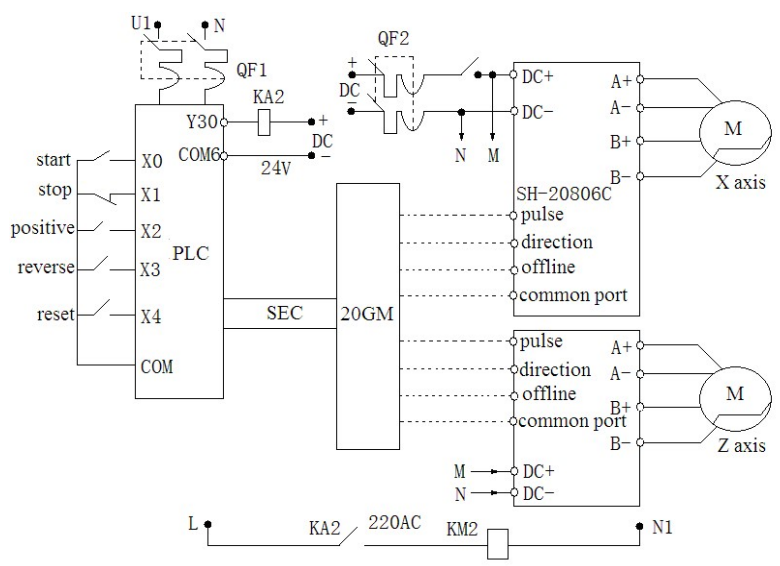

Figure 4 Connection of Two-axis Coordinated Control System

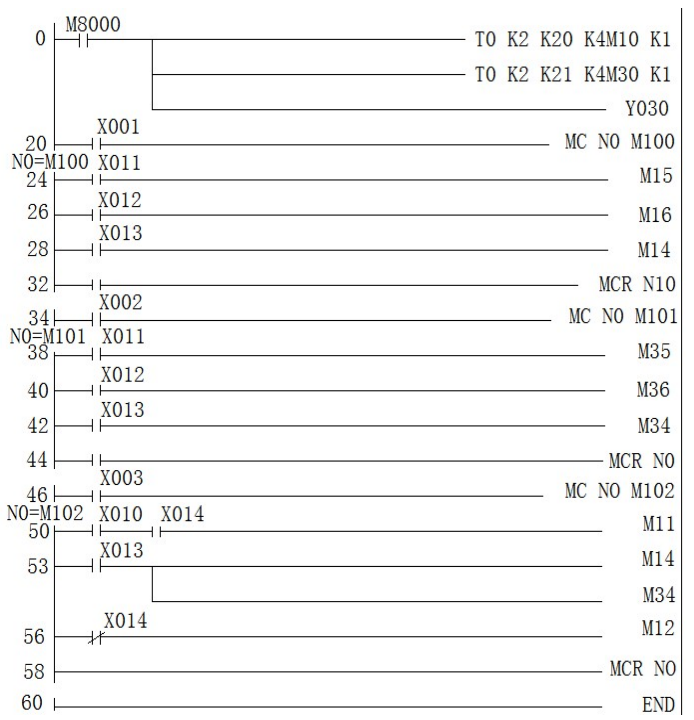

Figure 5 Program Example

\subsection{Design example}

As shown in figure 5, M80000 is in the power state when PLC is electric. The two TO instructions by M80000 control are writing the data command special function module and PLC module with special function is to buffer BFM write data command. Its meaning is When M80000 was electric, PLC K4M10, K4M11, K4M12, K4M25, and K4M30, K4M31, K4M32, K4M45 as the transfer source data to the special function of No. 2 unit in the module of BFM21. Because 20GM can make the motion of CNC machine tools into the buffer zone of the different position, the program only needs to be linked to the function of this position according to the different action mode. This greatly simplifies the logic relationship between programs, and provides great convenience for users ${ }^{[4]}$.

\subsection{External wiring}

As shown in figure 6. PLC, external wiring has the function of the completion of $\mathrm{X}, \mathrm{Z}$ two separate movements, linkage, movement and reset function, function distribution corresponding respectively:

(1) SA1, action selection; 
(2) SB1, start button of two-axis;

(3) SB5, stop button of two-axis;

(4) SB2, forward button;

(5) SB3, back button;

(6) SB4, reset button;

(7) KM, power switch of control contact.

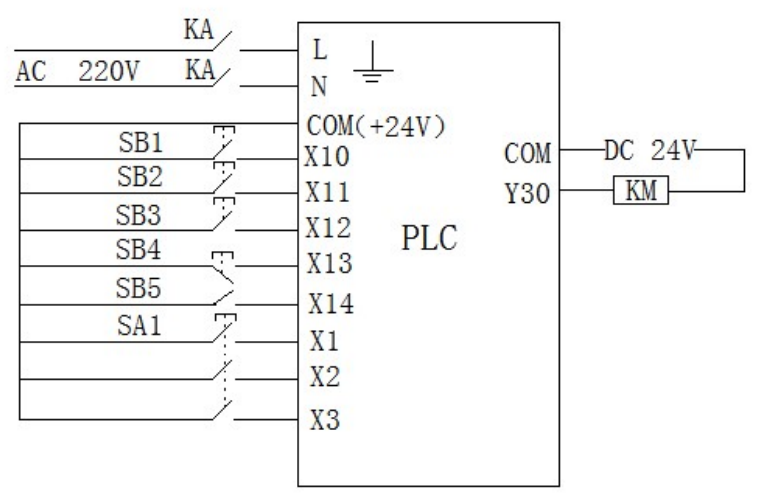

Figure 6 External Connection of PLC

\subsection{Design features}

\subsubsection{Simple program logic}

All axis actions of the CNC machine tool are included in each bit of the buffer due to the complete pertinence of the special module If one of the paths needs to be moved, all path processing will be completed to facilitate understanding of the procedure only the position must be placed.

\subsection{High portability}

The complete design of the program allows it to freely join other controlled programs of the $\mathrm{CNC}$ machine tool without affecting its own logical relationship and programming.

\subsubsection{Simulated multi-axis linkage NC machining environment}

According to the form of component curve, the multi-axis linkage NC machining environment can be simulated by mapping $\mathrm{CNC}$ machine tool, tool, fixture and machining process.

Comprehensive consideration, the design has the advantages of strong maintenance, high stability, and its application can greatly reduce production costs, and it is worth promoting and using.

\section{Conclusion}

This design scheme for complex curved surface parts has realized linkage function of $\mathrm{X}, \mathrm{Z}$ two- axis on $\mathrm{NC}$ machine tool, which is taking PLC as the core through the reasonable program design and the high motion control module. It can be extended to axis field and provides technical guarantee with certain scalability and practicability.

\section{References}

1. Congqian Qin. PLC technology and its application [M]. Beijing: Machinery Industry press, 2010.

2. Yu Cheng. Programmable control and programming skills [M]. Guangzhou: South China University of Technology press, 2011).

3. Chengxiao Liao. Numerical control technology [M]. Wuhan: Hubei Science and Technology press, 2011.

4. Chongwei Zhang. Motion control system [M]. Wuhan: Wuhan University of Technology press, 2010. 